\title{
33. Ultra-small-angle X-ray Scattering Study of the Structure of Colloidal Dispersions
}

\author{
By Hideki MatsuoKa, Koji KaKigami, and Norio IsE \\ Department of Polymer Chemistry, Kyoto University, Kyoto 606-01 \\ (Communicated by Seizo Okamura, M. J. A., Nov. 12, 1991)
}

\begin{abstract}
The ordered structure of polymethylmethacrylate-based latex particles in water dispersions have been studied by the Bonse-Hart type Ultra-small-angle X-ray scattering (USAXS) technique. The scattering patterns resembled a "powder diffraction pattern and distinct diffraction peaks were observed in the scattering curve. The lattice system was concluded to be a face-centered-cubic (fcc) lattice from the relative peak position. These findings indicate that the ordered structure has a limited size and that each ordered region orients randomly in the dispersion.
\end{abstract}

Key words: Ultra-small-angle X-ray scattering; latex dispersion; ordered structure; Bonse-Hart camera.

Introduction. In 1966, Bonse and Hart constructed an X-ray scattering apparatus having an extremely high small-angle resolution by using two grooved single crystals as a monochrometer and analyzer. ${ }^{1)}$ The measurable range was in the order of micrometers. They succeeded in measuring scattering profiles of latex powders of several sizes. This apparatus has a very high potentiality: (1) Application to turbid (e.g. colloidal dispersions) and opaque systems, which cannot be studied by light scattering (LS), is possible because of the Born approximation, (2) three-dimensional information can be obtained of the structure, and (3) the information obtained is for the whole structure, not just part of it. Although the Bonse-Hart camera has such advantages, this camera has been almost forgotten for a while except for a few examples ${ }^{2)}$ probably because of the difficulties of producing a goniometer with very high rotation accuracy, of obtaining perfect crystals and cutting them, and probably because the density fluctuation of such a large dimension (in the order of micrometers) did not attract attention.

In recent years, large-scale density fluctuations have drawn interest especially in the fields of colloid science, metal physics, polymer physics, etc. Microphase separations occur in polymer blend ${ }^{3)}$ and metal alloy systems. In latex dispersions at low ionic strengths, ordering phenomena, namely the submicron-order crystal-like three-dimensional arrangements of colloid particles in dispersion, take place due to the interparticle electrostatic interaction. ${ }^{4)}$ The ordered structure has been confirmed by ultramicroscopy. ${ }^{4}$ However, this technique still has some difficulties. First, the information of the structure obtained is two-dimensional, not three-dimensional. Secondly, the information is on a local, not global area or space. Thirdly, the observation is limited to a space which is close to the cell-glass surface. The information of the inside portion of the dispersion cannot be obtained. Some of these problems may be solved by LS. Unfortunately, LS is almost powerless for turbid systems like colloidal dispersions.

By small-angle scattering using X-rays as a light source, these problems would be solved. However, the conventional small-angle X-ray scattering (SAXS) apparatus has a small-angle limit up to about $1000 \AA$. This is acceptable for examining the ordering phenomena of ionic polymer solutions ${ }^{5)-9)}$ and micellar solutions, ${ }^{10)}$ but is too low for 
studying the density fluctuation in the micrometer order like the ordering phenomena of larger colloidal dispersion. The small-angle limit should be much higher.

For these reasons, we have constructed a Bonse-Hart type ultra-small-angle X-ray scattering (USAXS) apparatus, and succeeded to study the structure of polystyrene-based latex particles in water-ethanol dispersion, ${ }^{11), 12)}$ In the present study, we investigated the ordered structure of polymethylmethacrylate(PMMA)-based latex particles in water dispersions by the USAXS apparatus. PMMA particles facilitate measurements as will be mentioned below.

Experimental materials. The latices used were PMMA-based, MC-8 and MSC-14, which were synthesized ${ }^{13)}$ in our laboratory. PMMA latex particles have a higher electron density than polystyrene-based latex particles, which realizes a larger electron density difference between the scatterer and the medium (see Table I). This situation enables us to investigate the structure of water dispersions of latices much more easily with a shorter accumulation time. The diameter of the MC- 8 latex particle was $3000 \AA$ and the surface charge density was $1.9 \times 10^{-6} \mathrm{C} \mathrm{cm}^{-2}$. The latex dispersion was purified by ultrafiltration and ion-exchange resin (mixed-bed type, Biorad AG501-X8(D), Biorad Lab., Richmond, CA, USA). The measurements were performed within 2-3 hours, after the dispersion was pipetted into a USAXS cell to avoid the sedimentation effect, which may not be safely neglected for PMMA latex particles having a rather large specific gravity. Inside the USAXS cell, ion-exchange resin particles were added when the measurements were performed.

Table I. Electron density and specific gravity of particles and dispersion media

\begin{tabular}{ccc}
\hline Materials & $\begin{array}{c}\text { Electron density } \\
{\left[10^{23} \mathrm{~cm}^{-3}\right]}\end{array}$ & $\begin{array}{c}\text { Specific gravity } \\
{\left[\mathrm{g} \mathrm{cm}^{-3}\right]}\end{array}$ \\
\hline PMMA & 3.87 & 1.19 \\
PS & 3.40 & 1.05 \\
$\quad \begin{array}{l}\text { water } \\
\text { water-ethanol } \\
\text { mixture (3:2 in vol.) }\end{array}$ & 3.34 & 1.00 \\
\hline
\end{tabular}

PS: polystyrene-based latex.

USAXS apparatus. The details of the USAXS apparatus have been fully described elsewhere. ${ }^{12)}$ The USAXS apparatus was constructed by following the principle proposed by Bonse and Hart, which is quite different from conventional SAXS to cover the very small-angle regions. The upper limit of the Bragg distance of our apparatus was estimated to be about $8 \mu \mathrm{m}$ which is larger than that by Bonse and Hart (about $2 \mu \mathrm{m}$ ). The USAXS cell used in this study was the sandwich-type flat-cell instead of glass capillary which was used in our previous study ${ }^{11), 12)}$ to eliminate the influence of the total reflection of X-ray beam by the glass surface to the scattering curve. The polymer film used for the cell window was polyimide called Kapton of a thickness of $25 \mu \mathrm{m}$ because this film is strong enough to keep the sample in vacuum and has large chemical resistance. Two sheets of Kapton film (hence, totally $50 \mu \mathrm{m}$ thick) were put on both sides of a metal cell frame. The thickness of the sample was $2 \mathrm{~mm}$.

In this study, we made an additional effort to reduce background scattering. Since our USAXS optical system is set in a vacuum-tight chamber, some stray light reflected by the inside wall of the chamber may reach the detector (For a Bonse-Hart camera, it was not necessary to set up a slit just in front of the detector window to increase the resolution). 


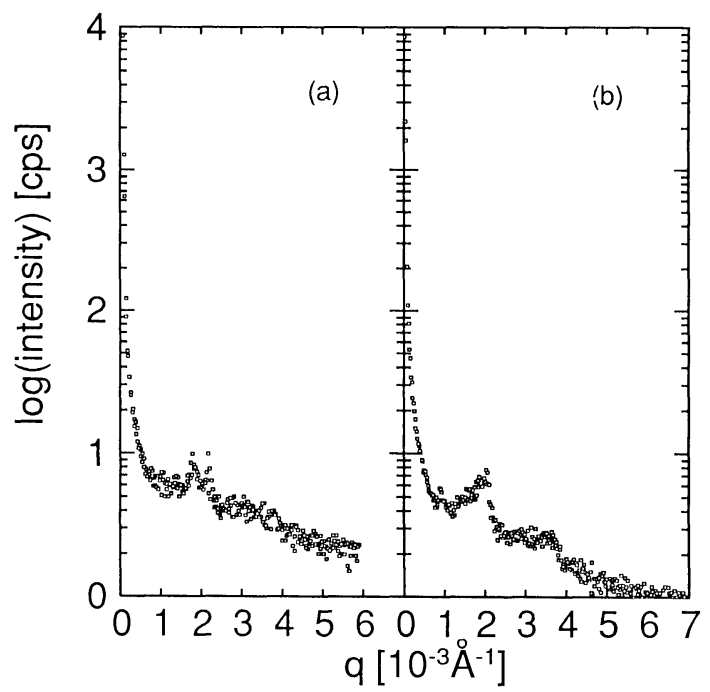

Fig. 1. USAXS curves of MSC-14 latex dispersion with and without $\mathrm{Pb}$ shields. (a) Without shields. Accumulation time $=30$ sec. (b) With shields. Accumulation time $=100$ sec. Particle diameter $=1200 \AA$. The abscissa, $q$, is a scattering vector $(=4 \pi \sin \theta / \lambda, 2 \theta$ : scattering angle, $\lambda$ : the wavelength of X-ray).

Thus, we set shields of lead at the back of the first crystal and around the detector. Fig. 1 shows examples of the USAXS pattern of latex dispersions (MSC-14, Diameter $=1200 \AA$, surface charge density: $3.0 \times 10^{-6} \mathrm{C} \mathrm{cm}^{-2}$, conc.: about 3.7 vol.\%) with and without the $\mathrm{Pb}$ shields. The background intensity was reduced to ca. $1 \mathrm{cps}$, which is nearly the limit of the proportional counter used as a detector, if we consider the electrical noise. The effect of the $\mathrm{Pb}$ shield is obvious. All measurements in this study were performed with the $\mathrm{Pb}$ shields.

Results and discussion. Fig. 2 shows the USAXS curves of water dispersions of the MC-8 latex at various concentrations. For each scattering curves except for the one at the lowest concentration $(1.4 \mathrm{vol} . \%)$ though not shown in the Figures, distinct diffraction peaks (indicated by thin arrow) were observed, which indicated the existence of an ordered structure of latex particles in dispersion. In addition, the scattering maxima (indicated by thick arrow) which were characteristic for an isolated sphere ${ }^{14)}$ were also observed. From the relative position of Bragg peaks, the lattice system of the dispersions can be concluded to be a face-centered cubic (fcc) lattice. (for fcc, the ratio of diffraction peak points should be $\sqrt{3}, \sqrt{4}, \sqrt{8}, \sqrt{11}, \sqrt{12, \ldots}{ }^{15)}$ ). For each scattering curve, the second peak is hardly observed, which is reflection from (200) plane, i.e., this is the second order peak. On the other hand, the first peak is from (111) plane, i.e., the first order peak. In addition, since the positions of these two peaks are very close, the second peak of an fcc structure is easy to merge into the first peak with increasing lattice distortion as has been clearly shown by theoretical calculation of the three-dimensional paracrystal model. ${ }^{16}$ )

The USAXS patterns shown in Fig. 2 resemble the "powder" diffraction pattern for a distorted fec crystal, not a "single crystal". It is often claimed that the "wall effect" plays an important role for the formation of ordered structure in latex dispersions, and that the structure is maintained only at the region close to the cell wall. The observation of powder-like pattern in Fig. 2 indicates that (1) the ordered structure is formed inside of the dispersion, not only in the region close to the cell wall, and (2) the ordered structures have 


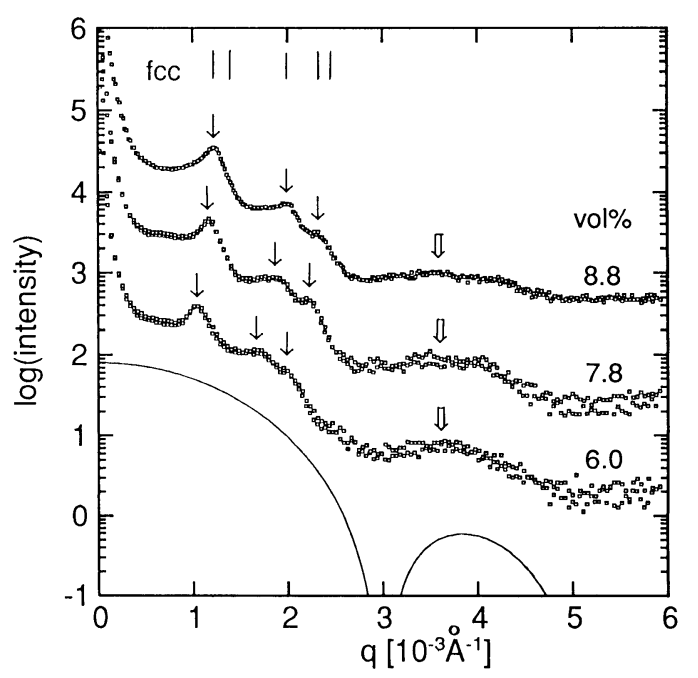

Fig. 2. USAXS curves of MC-8 latex dispersed in water at three concentrations. The solid line is a theoretical scattering curve of an isolated sphere of $3000 \AA$ in diameter. The vertical bars are peak positions expected for an fce structure for an $8.8 \mathrm{vol} . \%$ dispersion. Each scattering curve represents the superposition of data of positive and negative scattering angles.

a limited size and each ordered region orients randomly.

By microscopic observation, it is almost always confirmed that the (111) plane of fce structure is parallel to the cell glass surface. Sogami et al. observed the layered structures in the process of structure formation by laser Kossel line analysis. ${ }^{17)}$ In addition, in the process of colloidal crystal growth, although the (111) plane is formed in parallel to the cell glass surface, the interlayer distance was observed to be larger than that expected for an fec crystal. ${ }^{18)}$ From these observations, the wall-effect to the colloidal crystal growth could not be completely ruled out. However, no sign of the effect is seen, as far as the present USAXS data are concerned. ${ }^{19)}$

The scattering profile, I(q), contains information on the size and shape of the scatterer and on the spatial distribution of the scatterer. If the scatterer is spherical and monodisperse, $\mathrm{I}(\mathrm{q})$ is represented by the following simple equation;

$$
\mathrm{I}(\mathrm{q})=\mathrm{n} P(\mathrm{q}) \mathrm{S}(\mathrm{q}) \cdots(1)
$$

where $\mathrm{n}$ is the particle number, $\mathrm{P}(\mathrm{q})$ the particle factor, and $\mathrm{S}(\mathrm{q})$ the interparticle structure factor. $\mathrm{S}(\mathrm{q})$ reflects the spatial distribution of the center of mass of the scatterer. $\mathrm{P}(\mathrm{q})$ represents the single particle scattering. In this analysis, we equated the scattering profile at 1.4 vol.\% suspension to $\mathrm{P}(\mathrm{q})$. After the subtraction of the water scattering, the $\mathrm{S}(\mathrm{q})$ function was obtained by division of $\mathrm{I}(\mathrm{q})$ by $\mathrm{P}(\mathrm{q})$ after performing suitable normalization. An example is shown in Fig. 3.

The nearest interparticle distance $\left(2 \mathrm{D}_{\mathrm{exp}}\right)$ is calculated from the peak position $\left(\mathrm{q}_{\mathrm{m}}\right)$ of the $\mathrm{S}(\mathrm{q})$ function. For an fcc lattice, $2 \mathrm{D}_{\exp }$ is related to $\mathrm{q}_{\mathrm{m}}$ by the following equation;

$$
2 \mathrm{D}_{\exp }=\left(2 \pi / \mathrm{q}_{\mathrm{m}}\right)(\sqrt{3} / \sqrt{2}) \cdots(2) .
$$




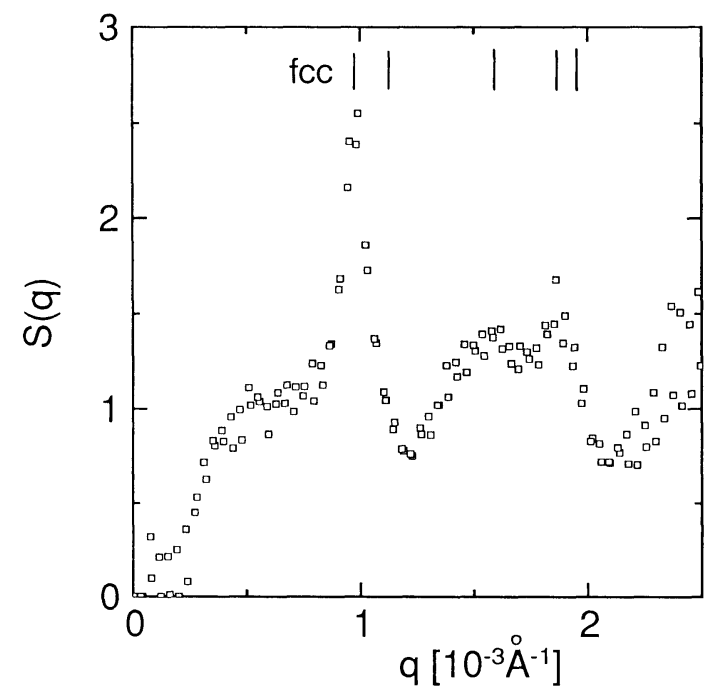

Fig. 3. Interparticle structure factor, $\mathrm{S}(\mathrm{q})$, of $\mathrm{MC}-8$ latex dispersed in water. Latex concentration : 4.3 vol. $\%$.

$2 \mathrm{D}_{\text {exp }}$ values thus estimated showed a satisfactory agreement with $2 \mathrm{D}_{0}$ values, the average interparticle distance calculated from the concentration. This is reasonable at the concentrations studied here (from 1.9 to 8.8 vol.\%). The tendency, $2 \mathrm{D}_{\text {exp }}<2 \mathrm{D}_{0}$, which indicates the existence of "two-state structure", should be observed in a much lower concentration range, although the $\mathrm{S} / \mathrm{N}$ ratio was not low enough for further study here.

In conclusion, the ordered structure, including the inside portion of the dispersion, of a PMMA-based latex in water dispersion could be studied by the USAXS technique. We confirmed that the ordered structure is maintained inside the dispersion and that the USAXS pattern is of the "powder" diffraction-type, which indicates that the ordered structures have a limited size. Further analysis, for example the comparison of $\mathrm{S}(\mathrm{q})$ function with paracrystal lattice factor $Z(q)$, is now in progress.

Acknowledgements. This study is supported by a grant-in-aid for specially promoted research of Ministry of Education, Science, and Culture (No. 63060003) to whom our sincere gratitude is due. Synthesis of the latices was kindly done by Mr. H. Yoshida of our laboratory.

\section{References}

1) U. Bonse, and M. Hart: Z. Phys., 189, 151 (1966).

2) D. Schwahn et al.: Nucl. Inst. Methods Phys. Res., A239, 229 (1985).

3) T. Hashimoto, M. Fujimura, and H. Kawai: Macromolecules, 13, 1660 (1980).

4) N. Ise: Angew. Chem., 25, 323 (1986).

5) N. Ise et al.: J. Amer. Chem. Soc., 101, 5836 (1979).

6) N. Ise et al:: ibid., 102, 7901 (1980).

7) N. Ise et al.: J. Chem. Phys., 81, 3294 (1984).

8) H. Matsuoka et al.: ibid., 83, 378 (1985).

9) Y. Yoshikawa, H. Matsuoka, and N. Ise: British Polym. J., 18, 242 (1986).

10) Y. Ishii, H. Matsuoka, and N. Ise: Ber. Bunsenges. Phys. Chem., 90, 50 (1986).

11) H. Matsuoka et al.: Proc. Natl. Acad. Sci. USA, 88, 6618 (1991).

12) H. Matsuoka, K. Kakigami, and N. Ise: The Rigaku Journal, 8, 8 (1991). 
13) H. Ono, and H. Saeki: Coll. Polym. Sci., 253, 744 (1975).

14) A. Guinier, and G. Fournet: Small-angle Scattering of X-rays. Wiley, New York (1955).

15) For example, B. D. Cullity: Elements of X-ray Diffraction. 2nd ed., Addison-Wesley (1978).

16) H. Matsuoka et al.: Phys. Rev. B, 36, 1754 (1987); H. Matsuoka et al., ibid., 41, 3854 (1990).

17) I. S. Sogami, and T. Yoshiyama: Phase Transitions, 21, 171 (1990).

18) H. Yoshida, K. Ito, and N. Ise: Phys. Rev. B, 44, 435 (1991).

19) Sometimes, we obtained USAXS profiles which could not be interpreted as "powder" diffraction: additional peaks were superimposed. This may be due to the effect of the cell wall or ordered structure may have grown up to a very large "single crystal", although the details are still not clear. 